\title{
Characterization and annotation of Babesia orientalis apicoplast genome
}

Yuan Huang ${ }^{1}$, Lan $\mathrm{He}^{1,2^{*}}$, Jinfang $\mathrm{Hu}^{1}$, Pei He ${ }^{1}$, Junwei $\mathrm{He}^{1}$, Long $\mathrm{Yu}^{1}$, Ngabu Malobi ${ }^{1}$, Yanqin Zhou ${ }^{1,2}$, Bang Shen ${ }^{1,2}$ and JunLong Zhao ${ }^{1,2^{*}}$

\begin{abstract}
Background: Babesia orientalis is an obligate intraerythrocytic protozoan parasite of the buffalo (Bubalus bubalis, Linnaeus, 1758) transmitted by the tick Rhipicephalus heamaphysaloides. It is the causative agent of water buffalo babesiosis, one of the most important pathogens of water buffalo in central and southern China. As a member of the phylum Apicomplexa, B. orientalis possesses a relatively independent and alga originated organelle the apicoplast. Apicoplasts in other apicomplexa parasites are involved in the biosynthesis of haem, fatty acids, iron-sulphur clusters and isoprenoids. Some of these metabolic pathways were shown to be essential for parasite survival, therefore can serve as potential drug targets.

Methods: 30 pairs of primers were designed based on the full genome sequence of B. orientalis (unpublished data) and by aligning reported apicoplast genomes of Babesia bovis and Theileria parva. Conventional PCRs was performed to obtain overlapped fragments to cover the whole apicoplast genome. Then the apicoplast genome of B.orientalis was sequenced, assembled and aligned with reported apicoplast genomes of B. bovis and T. parva. The obtained apicoplast genome was annotated by using Artemis and comparing with published apicomplexan apicoplast genomes. The SSU and LSU nucleotide sequences generated were used in a phylogenetic analysis using the maximum likelihood implemented in MAGE 6.0.
\end{abstract}

Results: We have obtained and analyzed the complete genome sequence of the $B$. orientalis apicoplast. It consisted of a $33.2 \mathrm{~kb}$ circular DNA (78.9\% A + T). The apicoplast genome unidirectionally encodes one large and one small subunit ribosomal RNAs, 24 tRNA genes, 4 DNA-dependent RNA polymerase beta subunits (rpoB, rpoC1, rpoC2a and rpoC2b), 17 ribosomal proteins, one EF-Tu elongation factor, 2 Clp protease chaperones, and 14 hypothetical proteins. In addition, it includes two copies of the $\mathrm{ClpC}$ gene. The structure and organization of the $B$. orientalis apicoplast genome are most similar to those of the B. bovis apicoplast.

Conclusions: This is the first report of the complete sequence of the $B$. orientalis apicoplast genome. This information should be useful in the development of safe and efficient treatment against buffalo babesiosis.

Keyword: Babesia orientalis, Apicoplast, Comparative analysis, Genome

\section{Background}

The protistan phylum Apicomplexa contains many species (e.g., Eimeria tenella, Plasmodium falciparum, Babesia bovis, Toxoplasma gondii, Cryptosporidium spp. and Cyclospora spp.) that are of great health and economic concerns. Most apicomplexan parasites, with the exception of Cryptosporidium spp. and Gregarina spp. [1, 2],

\footnotetext{
* Correspondence: helan@mail.hzau.edu.cn; zhaojunlong@mail.hzau.edu.cn 'State Key Laboratory of Agricultural Microbiology, College of Veterinary Medicine, Huazhong Agricultural University, Wuhan 430070, Hubei, P.R. China Full list of author information is available at the end of the article
}

have a relict, non-photosynthetic plastid called the apicoplast $[3,4]$. The apicoplast was acquired by secondary endosymbiosis from a eukaryotic alga (but it is still under debate whether it is from a red or a green alga) $[5,6]$. It is involved in critical metabolic pathways such as the synthesis of haem, fatty acids, iron-sulphur clusters and isoprenoids. Some of these metabolic pathways are essential for parasite survival and are considered as potential targets for anti-parasitic drug designs. It was reported that the apicoplast housekeeping machinery, specifically apicoplast DNA replication, transcription and translation, was 
targeted by ciprofloxacin, thiostrepton and rifampin, respectively [7].

Like mitochondria, the apicoplast possesses its own genome. Thus, the complete apicoplast genomes of several apicomplexan parasites have been characterized, which include Plasmodium spp., Leucocytozoon caulleryi, Theileria parva, T. gondii, E. tenella, Cyclospora cayetanensis and B. bovis [8-15]. The entire mitochondria genome of $B$. orientalis has been characterized and the phylogenetic analysis has revealed that $B$. orientalis belong to the Babesia clade with $B$. bovis as the closest relationship [16]. However, there is no report on the apicoplast genome of $B$. orientalis.

$B$. orientalis is an intra-erythrocytic protozoan parasite which causes babesiosis with clinical manifestation of fever, anemia, icterus, haemoglobinuria and high mortality in water buffalo. $B$. orientalis differs from $B$. bigemina and $B$. bovis in transmission vectors, morphology, pathogenicity and characteristics of in vivo cultivation. It causes significant economic losses in central and south China $[17,18]$. Recent work focuses on gene diversity, metabolism process, pathogenicity aspect and identification of new markers to improve the diagnosis and therapy of buffalo babesiosis.

In this study, the full sequence of the $B$. orientalis (Wuhan strain) apicoplast genome was determined, annotated and characterized. This is the first report of the complete nucleotide sequence of the $B$. orientalis apicoplast genome. The data generated contribute to the prevention and control of buffalo babesiosis.

\section{Methods}

\section{Parasites and animal experiments}

Blood samples were collected from water buffalo that were experimentally infected with $B$. orientalis in Huazhong Agricultural University [19]. Genomic DNA was extracted using the QIAamp DNA Blood Mini Kit (Qiagen, Hilden, Germany), according to the manufacturer's instructions.

\section{Ethics statement}

Experimental animals were housed, fed and given clean drinking water according to the stipulated rules for experimental usage of laboratory animals (the regulation of the administration of affairs concerning experimental animals of P.R. China). All protocols were approved by the Laboratory Animal Research Centre of Hubei province, and the ethical committee of Huazhong Agricultural University (permit number 4200696657).

\section{Cloning and sequencing of $B$. orientalis apicoplast genome}

Partial sequences of the $B$. orientalis apicoplast were initially obtained from a high-throughput whole genome sequencing project (data unpublished). To obtain the fulllength sequence of the apicoplast genome and fill the gaps between fragments, we designed primers (Additional file 1: Table S1) based on the apicoplast partial sequences. Conventional PCRs was performed to obtain overlapped fragments to cover the whole apicoplast genome. The PCR amplicons were subjected to sequencing directly on an ABI3700 Autosequencer (Applied Biosystems, Foster City, $\mathrm{CA}$ ) or cloned into pMD19-T vector (TaKaRa Biotechnology) and sequenced subsequently.

\section{Sequence analysis and annotation}

The Software Artemis [20, 21] was used in the annotation of the $B$. orientalis apicoplast genome. The entire apicoplast genome of $B$. orientalis (GenBank accession no. KT428643) was scanned for potential open reading frames (ORFs). The putative coding regions were conceptually translated and annotated using the published P. falciparum and B. bovis apicoplast genomes as references. These annotations were further refined by BLAST (http://blast.ncbi.nlm.nih.gov/Blast.cgi) searches against the GenBank database. The small and large subunits of rRNA genes (SSU and LSU rRNA, respectively) were determined by comparing with their counterparts in T. parva and B. bovis. The tRNA genes were annotated by using tRNAscan-SE server (http://lowelab.ucsc.ucsc.edu/tRNAscan-SE/) with the Mito/Chloroplast model and the Nematode Mito model [22]. The online software CGView (http://stothard.afns.ualberta.ca/cgview_server) [23] was used in generating the genetic maps. The reference genomes used included: AAXT01000007 (B. bovis), HM222968 (Chromera CCMP3155), fusion of X95275 (IRA) and X95276 (IRB) (P. falciparum), NC_004823 (E. tenella), AAGK01000009 (T. parva) and U87145 (T. gondii). Phylogenetic analysis of the SSU and LSU genes was performed using the Maximum Likelihood method implemented in MEGA 6.0 (http://www.megasoftware.net) [24]. The transmembrane domains and functional domains were predicted using TMpred (http:// www.ch.embnet.org/software/TMPRED_form.html) [25] and Pfam (http://pfam.sanger.ac.uk/) [26].

\section{Results and discussion}

\section{Characterization of the circular apicoplast genome of B. orientalis}

Previous efforts aimed to determine the full genome sequence of $B$. orientalis (unpublished data) has found several contigs containing putative apicoplast genome sequences of this parasite. In order to identify the full length sequence of the apicoplast genome, 30 pairs of PCR specific primers based on the sequence information from the aforementioned contigs were designed to obtain amplicons covering the complete apicoplast 
genome. Sequencing and assembly of these amplicons have shown that the apicoplast genome of $B$. orientalis is a $33.2 \mathrm{kbp}$ circular DNA with a high $\mathrm{A}+\mathrm{T}$ content of $78.97 \%$, similar to that of B. bovis (33.3 kbp, $\mathrm{A}+\mathrm{T} \%=78.2 \%)$.

Bioinformatic analysis indicated that the circular DNA contains 1 LSU rRNA gene, 1 SSU rRNA gene, 38 protein-coding ORFs, and 24 tRNA genes (Table 1). The 38 ORF genes include four DNA-dependent RNA polymerase beta subunits (rpoB, rpoC1, rpoC2a and rpoC2b), 17 ribosomal proteins, and one EF-Tu elongation factor (TufA), 2 Clp protease chaperone genes, 14 hypothetical proteins ranging in size from 30 (Hyp-11) to 193 amino acids (Hyp-8) (Fig. 1 and Table 1). The sufB gene coding for a protein involved in the assembly of iron-sulfur clusters in Plasmodium falciparum [14] apicoplast genome was not found in this genome. The content and arrangement of genes in the $B$. orientalis apicoplast genome are more similar to those of B. bovis [10] and T. parva [11] than those of P. falciparum [27] and T. gondii [28]. All genes encoded in the apicoplast genome of $B$. orientalis are transcribed in the same orientation and from the same strand (Fig. 1 and Table 1).

The majority of the coding sequences of proteins in the apicoplast genome of $B$. orientalis are not overlapped, except for three genes, which were found to overlap over one to three codons. Twenty-four tRNA genes were identified in the B. orientalis apicoplast genome, which are the identical number and type in $T$. gondii, $T$. parva and $B$. bovis. The tRNA gene Leu"-UAA has an intron, and is conserved among these species as well.

The $B$. orientalis apicoplast genome has ribosomal proteins and RNAs very similar to those of other apicomplexans. The genome analysis revealed proteins for the large (7 rpl proteins) small (10 rps proteins) ribosomal subunits. Other ribosomal proteins are encoded by nuclear genome

Table 1 Gene contents of the B.orientalis apicoplast genome

\begin{tabular}{|c|c|}
\hline Class & Genes \\
\hline Ribosomal RNA & $16 \mathrm{~S}, 23 \mathrm{~S}$ \\
\hline \multirow[t]{3}{*}{ Transfer RNA ${ }^{a, b}$} & $\begin{array}{l}A^{U G C}, C^{G C A}, D^{G U C}, E^{U U C}, F^{G A A}, G^{U C C}, H^{G U G}, \\
I^{G A U}, K^{\cup U U}, L^{\cup A G}, L^{U A A^{*}}\end{array}$ \\
\hline & $\begin{array}{l}M^{C A U}, M^{C A U}, N^{G U U}, P^{\mathrm{UGG}}, Q^{U U G}, R^{U C U}, R^{A C G}, \\
S^{G C U}, S^{U G A}, T^{\cup G U}, V^{U A C}\end{array}$ \\
\hline & $W^{C C A}, Y^{G U A}$ \\
\hline \multirow[t]{2}{*}{ Ribosomal proteins } & $\operatorname{rps} 2,3,4,5,7,8,11,12,17,19$ \\
\hline & rpl2, 4, 5, 6, 14, 16, 36 \\
\hline RNA polymerase & rpoB, rpoC1, rpoC2a, rpoC2b \\
\hline Other proteins & $\mathrm{clpC1}, \mathrm{clpC2}$, tufA \\
\hline Unassigned ORFs & 14 ORFs (hyp1-14) \\
\hline
\end{tabular}

${ }^{a}$ Single letter amino acid code and anti-codon

${ }^{\mathrm{b}}$ Indicating intron-containing genes and targeted to the apicoplast according to the analysis of the full genome of $B$. orientalis (unpublished data). Thus ribosomal proteins encoded by the apicoplast and nuclear genomes with $16 \mathrm{~S}$ and 23S-like rRNA are part of the apiRibosome of the $B$. orientalis. However we did not observe any $5 \mathrm{~S}$ ribosomal RNA encoding rff gene in the $B$. orientalis apicoplast genome, which indicates that either the apicoplast ribosomes in this species are free of 5S rRNA regulation or the apicoplast can import $5 \mathrm{~S}$ from the cytoplasm (as shown previously for the mammalian mitochondria [29]). Alternatively, it expresses a highly divergent $\mathrm{rff}$ gene. In this perspective, it is noteworthy that although the chloroplast genome of Chromera has an rff gene, no rff genes can be observed in the apicoplast genome of any apicomplexan parasite sequences so far.

The apicoplast genome of $B$. orientalis has 14 hypothetical coding sequences (hyp1-14). Hyp2-7 are the same ORF but repeat 6 times (Figs. 1 and 2). Some proteins show significant homology to other proteins in available databases but do not have any recognizable functional domains. Other apicomplexan parasites have similar hypothetical proteins in the same genomic regions. Corresponding region in $B$. bovis also exists several same ORF (Hyp 320-280) but repeat only 5 times (Fig. 2). Further work is needed to determine whether these CDs are expressed or are artifacts of annotation.

\section{B. orientalis carries an apicoplast genome similar to B. bovis}

To determine the synteny of $B$. orientalis apicoplast genome with other apicomplexan parasites, we compared the gene arrangements in four gene clusters (Fig. 1) from the $B$. orientalis apicoplast genome with the same gene clusters found in other apicomplexan parasites as well as the chloroplast genomes of Chromera algae [6]. Genes that encode ribosomal proteins and EF-Tu elongation factor are included in cluster 1. As shown in Fig. 2, the apicoplast genome of $B$. orientalis has a gene organization in Cluster 1 region identical to their counterparts in Babesia bovis and Theileria parva, of which remarkably all lack the rpl23 gene found in Chromera and Plasmodium species. Additionally the rps 13 gene is located between rps5 and rpl36 in Chromera sp., whereas that region in most apicoplast genomes, contain an uncharacterized gene or no coding sequence in the case of T. gondii and E. tenella (Fig. 2). A hypothetical protein (Hyp-1) is found in the same region of $B$. orientalis. Similar to $B$. bovis, there are several putative duplicated CDSs (Hyp2-Hyp7) following rps7 (Fig. 2). In addition, the duplicated CDSs found in B. orientalis have high homology to those in B. bovis.

Nine tRNA genes in Cluster 4 of the $B$. orientalis are adjacent to Cluster 1, meanwhile 5 tRNA genes in 


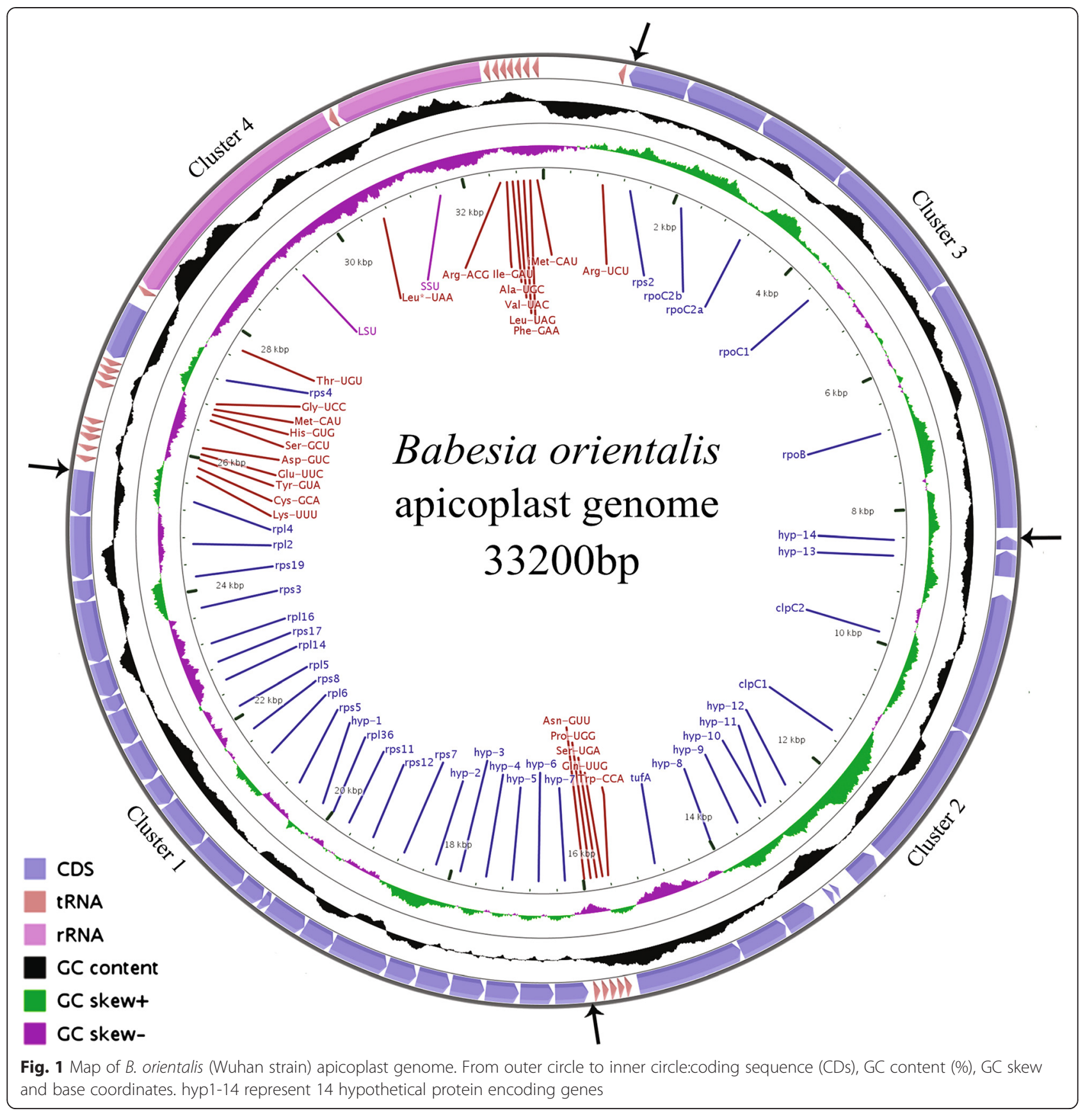

Cluster 2 are adjacent to Cluster 1 (Fig. 3). One additional tRNA genes for Asn (GUU) is absent in $T$. parva but present between the junction region of Cluster 1 and Cluster 2 in B. orientalis and B. bovis. Like $T$. parva and $B$. bovis, the junction between Cluster 1 and Cluster 2 in $B$. orientalis has several uncharacterized coding sequences without any clear functions, some of which appear to be identical copies derivated from a duplication process (Boh9 and Boh12). The duplicated CDSs found in B. orientalis do not share homology to duplicated sequences present in B. bovis and T. parva (Fig. 4). Based on these observations, sequences in the area between cluster 1 and cluster 2 is most likely the recombination event region, which probably has played an important role in the evolution of piroplasmida (Figs. 3 and 4).

ClpC chaperones genes are found in cluster 2 (Fig. 5), have a significant similarity to their orthologs in $B$. bovis and $T$. parva, and are subjected to a duplication process with 2 copies of the AAA_ 2 ATPase domain in B. orientalis (Figs. 3 and 4). Two hypothetical proteins (hyp-13 


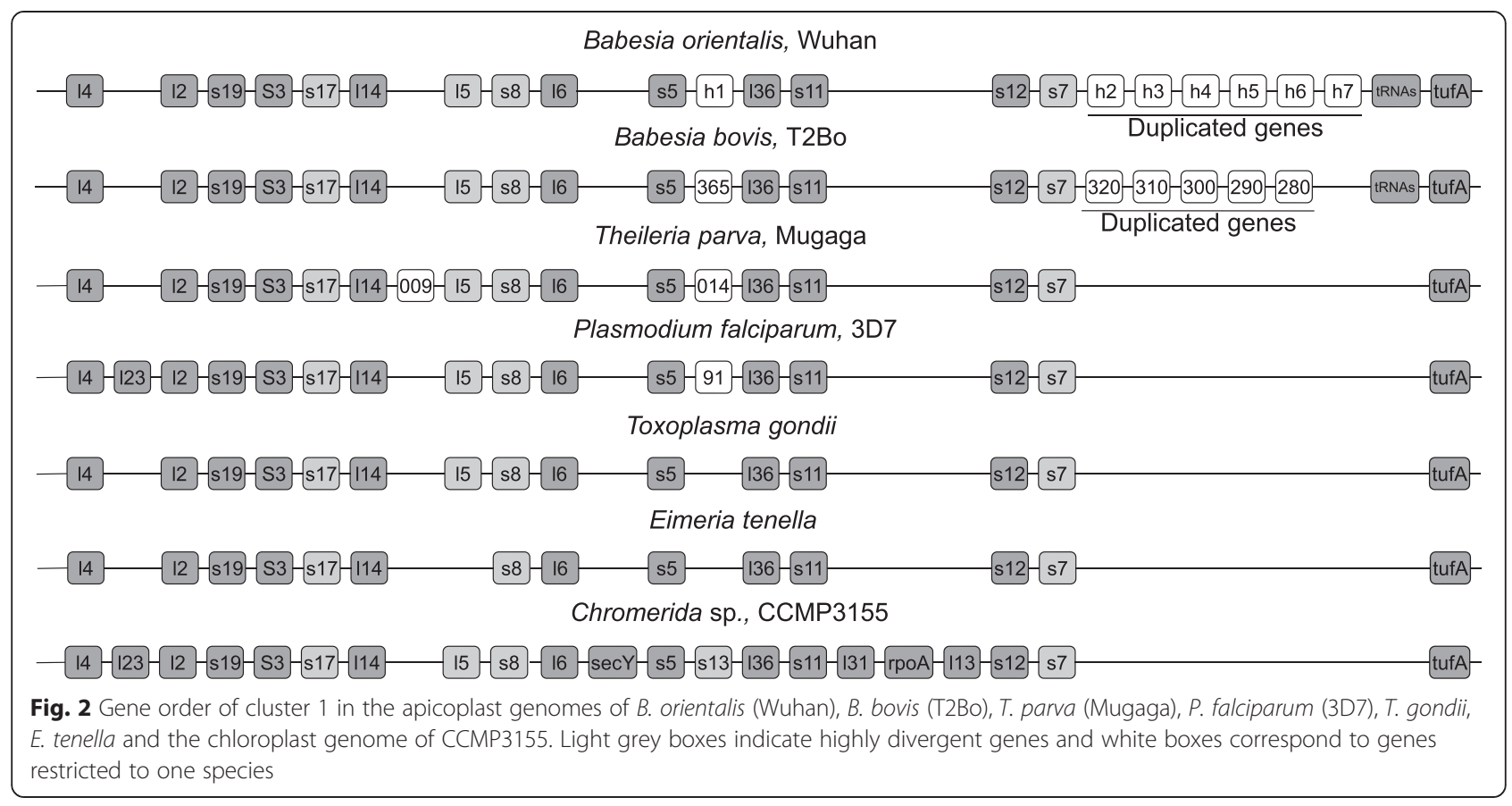

and hyp-14) belong to the area of cluster 2 adjacent to cluster 3 (Figs. 3 and 4).

Similar to B. bovis, P. falciparum and T. parva, we did not observe any rpl11 ribosomal gene in the $B$. orientalis apicoplast genome (Figs. 2 and 5). Thus, B. orientalis ribosomes do not need any L11 protein, or an rpl11-like protein encoded in nuclear genome level is actively involved during the process of translation, which is fundamentally different from that showed in $T$. gondii and prokaryotes. Conservation in the order of rpl11-clpC has been reported in Chromera and T. gondii (Fig. 4), whereas there is a lack of the rpl11 gene in plastid genomes of Aconoidasida, which have shown a rearrangement of the tRNA region adjacent to the clpC gene.

In cluster 3 of the $B$. orientalis apicoplast genome, there are RNA polymerases genes $(\mathrm{rpoB}, \mathrm{rpoC} 2 \mathrm{a}$ and
rpoC2b) and an rps2 gene that encodes S2 ribosomal protein (Fig. 1). Gene content and orientation of Cluster 3 genes in B. orientalis, T. parva and B. bovis is almost the same, suggesting it is a conserved region during the evolution of piroplasmida.

Ribosomal DNA genes are found in Cluster 4, especially a single set of SSU and LSU genes. However, a clear divergence among apicomplexans species regarding gene order and content is observed. In Chromera, SSU and LSU separated by a putative coding sequence, have the same orientation. In contrast, the orientation in Toxoplasma gondii and Plasmodium species is reversed, whereas in B. orientalis, B. bovis and T. parva, the 2 genes are transcribed in the same direction. Like $B$. bovis and T. parva, the Leu"-UAA tRNA gene with an intron is located between the SSU and LSU genes in B. orientalis (Fig. 5). Phylogenetic analysis based on the sequences 


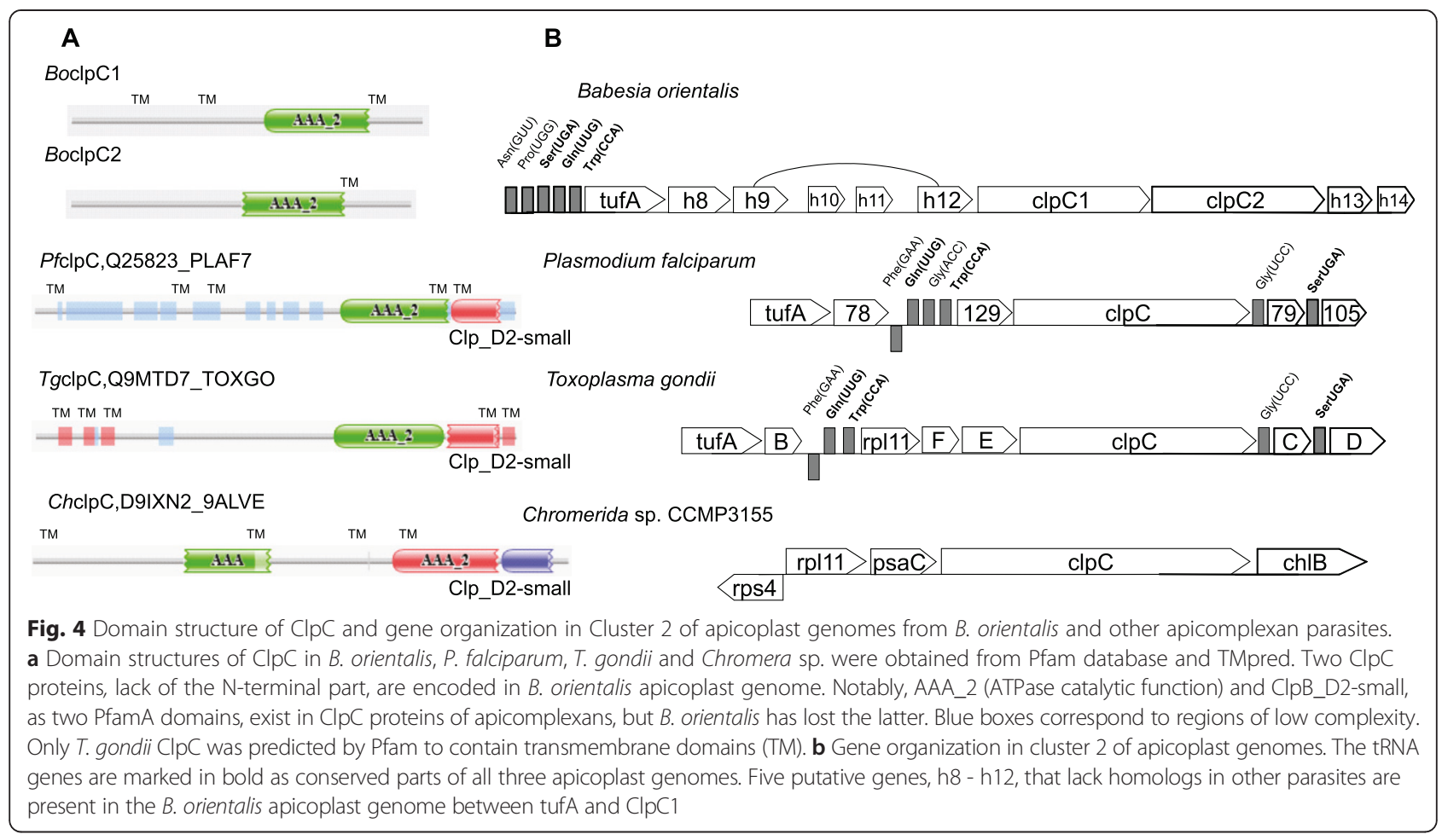

of SSU and LSU confirmed a close relationship between B. orientalis and B. bovis (Fig. 5).

Comparative analysis of the apicoplast genomes reveals that rearrangements have occurred over different stages of evolution in the apicoplast establishment, and the loss of genes involved in the photosynthesis physiological pathway has contributed to the apicoplast evolution process. RNA polymerase region inversion, lack of sufB gene, rearrangement of rRNA locus and duplication of clpC gene to form different paralogs are major events in early evolution process of piroplasma organism. The structure modification in

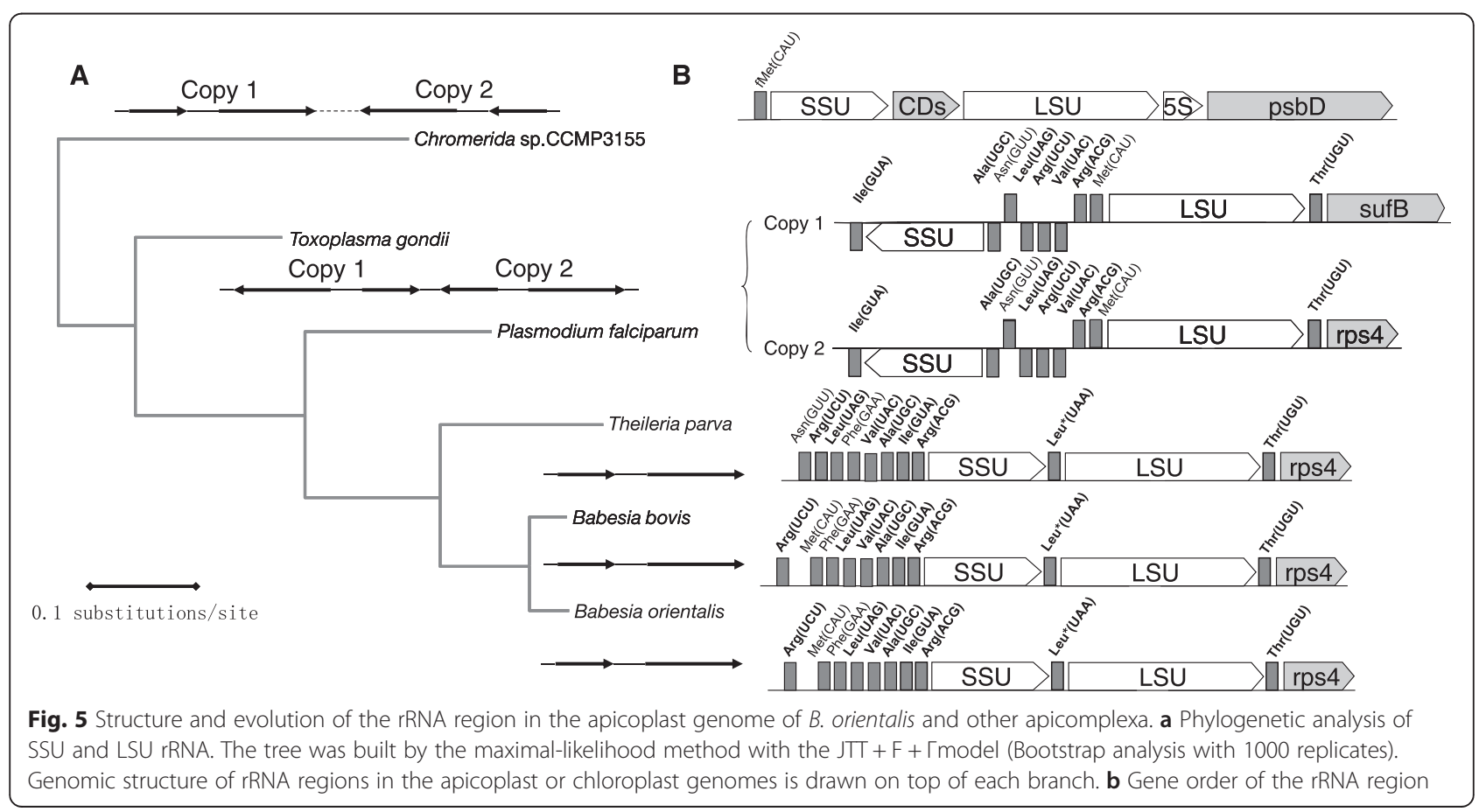


the $B$. orientalis apicoplast genome and DNA expansion (duplication of small region), similar to these in B. bovis, may be parts of the core apicoplast genome organization in apicomplexans. The only difference of apicoplast genome between $B$. orientalis and $B$. bovis exists in the two regions of duplicated genes in Cluster 1 and Cluster 2 (Figs. 1, 2 and 3).

\section{Conclusion}

In this study, the full $B$. orientalis apicoplast genome was sequenced and annotated. This analysis indicates that the $B$. orientalis apicoplast genome is more similar to that of B. bovis and T. parva than that of P. palciparum, E. tenella and T. gondii. Complete annotation of the organelle genome indicates that almost all genes identified in the B. bovis plastid genome are also present in this parasite. Our studies revealed that this $33.2 \mathrm{~kb}$ circular genome encodes machineries involved in gene transcription and translation within this organelle. The genes with metabolic functions are largely absent, although 14 small hypothetical proteins without specified functions need further studies. Our analysis of the apicoplast genome of $B$. orientalis complements our prior annotation of the mitochondria genome of this pathogen [16]. To improve our understanding of the physiological pathway in the apicoplast genome, we are currently trying to identify structural and regulatory proteins that are encoded by the nuclear genome but targeted to the apicoplast. Due to the functional importance of the apicoplast, the information collected from this study contributes to the understanding of metabolism in $B$. orientalis such as the isoprenoid biosynthesis. The potential parasiticidal activities of azithromycin and clindamycin [7] indicate other drug with putative targets in the apicoplast genome can be developed for the treatment of $B$. orientalis infection. Thus, our knowledge of the structural organization of the apicoplast genome of $B$. orientalis will likely facilitate the development of efficient therapy against buffalo babesiosis.

\section{Additional file}

Additional file 1: Table S1. PCR primers used in sequencing the Babesia orientalis apicoplast genome. (DOC 67 kb)

\section{Competing interests}

The authors declare that they have no competing interests.

\section{Authors' contributions}

All authors contributed actively to this study. LH and JLZ designed the experiments, $\mathrm{YH}$ carried out field and laboratory experiments and analyzed the data. JFH, PH, JWH, LY, WW, Malobi and YQZ participated in animal experiments and laboratory work. YH, LH, BS and JLZ wrote the paper. All authors read and approved the manuscript.

\section{Acknowledgements}

This study was supported by the National Key Basic Research Program (973 program) of China (Grant No. 2015CB150302), the National Natural Science Foundation of China (31302082), Huazhong Agricultural University Scientific \& Technological Self-innovation Foundation (Program No.2662014BQ020) and the Fundamental Research Funds for the Central Universities (2662015PY048).

\section{Author details}

${ }^{1}$ State Key Laboratory of Agricultural Microbiology, College of Veterinary Medicine, Huazhong Agricultural University, Wuhan 430070, Hubei, P.R. China. ${ }^{2}$ Key Laboratory of Animal Epidemical Disease and Infectious Zoonoses, Ministry of Agriculture, Huazhong Agricultural University, Wuhan 430070, Hubei, China.

Received: 11 September 2015 Accepted: 8 October 2015

Published online: 16 October 2015

\section{References}

1. Zhu G, Marchewka MJ, Keithly JS. Cryptosporidium parvum appears to lack a plastid genome. Microbiology. 2000;146(Pt 2):315-21.

2. Toso MA, Omoto CK. Gregarina niphandrodes may lack both a plastid genome and organelle. J Eukaryot Microbiol. 2007;54(1):66-72.

3. Foth BJ, McFadden Gl. The apicoplast: a plastid in Plasmodium falciparum and other Apicomplexan parasites. Int Rev Cytol. 2003;224:57-110.

4. McFadden Gl. The apicoplast. Protoplasma. 2011;248(4):641-50.

5. Kohler S, Delwiche CF, Denny PW, Tilney LG, Webster P, Wilson RJ, et al. A plastid of probable green algal origin in Apicomplexan parasites. Science. 1997;275(5305):1485-9.

6. Janouskovec J, Horak A, Obornik M, Lukes J, Keeling PJ. A common red algal origin of the apicomplexan, dinoflagellate, and heterokont plastids. Proc Natl Acad Sci U S A. 2010;107(24):10949-54.

7. Aboulaila M, Munkhjargal T, Sivakumar T, Ueno A, Nakano Y, Yokoyama M, et al. Apicoplast-targeting antibacterials inhibit the growth of Babesia parasites. Antimicrob Agents Chemother. 2012;56(6):3196-206.

8. Imura T, Sato S, Sato Y, Sakamoto D, Isobe T, Murata K, et al. The apicoplast genome of Leucocytozoon caulleryi, a pathogenic apicomplexan parasite of the chicken. Parasitol Res. 2014;113(3):823-8.

9. Lau AO, McElwain TF, Brayton KA, Knowles DP, Roalson EH. Babesia bovis: a comprehensive phylogenetic analysis of plastid-encoded genes supports green algal origin of apicoplasts. Exp Parasitol. 2009;123(3):236-43.

10. Brayton KA, Lau AO, Herndon DR, Hannick L, Kappmeyer LS, Berens SJ, et al. Genome sequence of Babesia bovis and comparative analysis of apicomplexan hemoprotozoa. PLoS Pathog. 2007;3(10):1401-13.

11. Gardner MJ, Bishop R, Shah T, de Villiers EP, Carlton JM, Hall N, et al. Genome sequence of Theileria parva, a bovine pathogen that transforms lymphocytes. Science. 2005;309(5731):134-7.

12. Cai $X$, Fuller AL, McDougald LR, Zhu G. Apicoplast genome of the coccidian Eimeria tenella. Gene. 2003;321:39-46.

13. Williamson DH, Denny PW, Moore PW, Sato S, McCready S, Wilson RJ. The in vivo conformation of the plastid DNA of Toxoplasma gondii: implications for replication. J Mol Biol. 2001;306(2):159-68.

14. Wilson RJ, Denny PW, Preiser PR, Rangachari K, Roberts K, Roy A, et al. Complete gene map of the plastid-like DNA of the malaria parasite Plasmodium falciparum. J Mol Biol. 1996;261(2):155-72.

15. Tang K, Guo Y, Zhang L, Rowe LA, Roellig DM, Frace MA, et al. Genetic similarities between Cyclospora cayetanensis and cecum-infecting avian Eimeria spp. in apicoplast and mitochondrial genomes. Parasites Vectors. 2015; 8(1)

16. He L, Zhang Y, Zhang QL, Zhang WJ, Feng HH, Khan MK, et al. Mitochondrial genome of Babesia orientalis, apicomplexan parasite of water buffalo (Bubalus babalis, Linnaeus, 1758) endemic in China. Parasite Vector. 2014;7:82.

17. Liu Q, Zhao JL, Zhou YQ, Liu EY, Yao BA, Fu Y. Study on some molecular characterization of Babesia orientalis. Vet Parasitol. 2005;130(3-4):191-8.

18. He L, Zhou YQ, Oosthuizen MC, Zhao JL. Loop-mediated isothermal amplification (LAMP) detection of Babesia orientalis in water buffalo (Bubalus babalis, Linnaeus, 1758) in China. Vet Parasitol. 2009;165(1-2):36-40.

19. He L, Feng HH, Zhang QL, Zhang WJ, Khan MK, Hu M, et al. Development and evaluation of real-time Pcr assay for the detection of Babesia Orientalis 
in water buffalo (Bubalus Bubalis, Linnaeus, 1758). J Parasitol. 2011;97(6):1166-9.

20. Rutherford K, Parkhill J, Crook J, Horsnell T, Rice P, Rajandream MA, et al. Artemis: sequence visualization and annotation. Bioinformatics. 2000;16(10):944-5.

21. Carver T, Harris SR, Berriman M, Parkhill J, McQuillan JA. Artemis: an integrated platform for visualization and analysis of high-throughput sequence-based experimental data. Bioinformatics. 2012;28(4):464-9.

22. Schattner P, Brooks AN, Lowe TM. The tRNAscan-SE, snoscan and snoGPS web servers for the detection of tRNAs and snoRNAs. Nucleic Acids Res. 2005; 33(Web Server issue):W686-689.

23. Stothard $\mathrm{P}$, Wishart DS. Circular genome visualization and exploration using CGView. Bioinformatics. 2005;21(4):537-9.

24. Tamura K, Dudley J, Nei M, Kumar S. MEGA4: molecular evolutionary genetics analysis (MEGA) software version 4.0. Mol Biol Evol. 2007;24(8):1596-9.

25. Hofmann KSW. TMbase - a database of membrane spanning proteins segments. Biol Chem. 1993;374:166.

26. Punta M, Coggill PC, Eberhardt RY, Mistry J, Tate J, Boursnell C, et al. The Pfam protein families database. Nucleic Acids Res. 2012;40(D1):D290-301.

27. Arisue N, Hashimoto T, Mitsui H, Palacpac NM, Kaneko A, Kawai S, et al. The Plasmodium apicoplast genome: conserved structure and close relationship of P. ovale to rodent malaria parasites. Mol Biol Evol. 2012;29(9):2095-9.

28. Denny P, Preiser P, Williamson D, Wilson I. Evidence for a single origin of the $35 \mathrm{~kb}$ plastid DNA in apicomplexans. Protist. 1998;149(1):51-9.

29. Magalhaes PJ, Andreu AL, Schon EA. Evidence for the presence of $5 S$ rRNA in mammalian mitochondria. Mol Biol Cell. 1998;9(9):2375-82.

\section{Submit your next manuscript to BioMed Central and take full advantage of:}

- Convenient online submission

- Thorough peer review

- No space constraints or color figure charges

- Immediate publication on acceptance

- Inclusion in PubMed, CAS, Scopus and Google Scholar

- Research which is freely available for redistribution 